\title{
DECIMETRIC REVERSE DRIFT AND U-TYPE BURSTS IN THE APRIL 9, 2001 FLARE
}

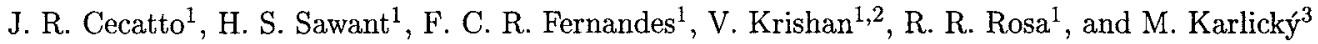 \\ ${ }^{1}$ National Institute for Space Research - INPE, São José dos Campos, SP - Brazil \\ ${ }^{2}$ Indian Institute of Astrophysics, Bangalore, India \\ ${ }^{3}$ Ondř ejov Observatory, 25165 Ondřejov, Czech Republic
}

\begin{abstract}
The Brazilian Solar Spectroscope (BSS) as well as the radio spectrographs of the Ondrejov Observatory (OO) observed, in decimeter wavelengths, spectra consisting of groups of type III reverse drift (RS) bursts with time resolution of $50 \mathrm{~ms}$ and also observed a partial inverted long duration (about $25 \mathrm{~s}$ ) U-type burst. The speeds of their exciting agents have been determined. The estimated speeds suggest that distinct exciters are responsible for the emissions of the inverted U-type and type-III RS bursts.
\end{abstract}

\section{OBSERVATIONS AND DISCUSSION}

High-resolution observations of the solar decimetric radio emission are of a great diagnostic value for understanding particle acceleration and the mechanism of energy release in flares. Time variations, periodic or otherwise, are related to the process of energy release. Here, we report for the first time high resolution (50 ms) observations of decimetric type-III RS bursts. Also, a partial inverted U-type burst of long duration (25 s) is being reported for the first time in the frequency range of 900-2000 MHz. This flare has also been observed by the SOHO spacecraft, and the SXT instrument on the Yohkoh satellite. Details of the spacecraft observations will be published elsewhere.

The spectra recorded at the OO show a U-type like burst starting at 15:25:23 UT around $1000 \mathrm{MHz}$. This burst attains its minimum mean frequency of $900 \mathrm{MHz}$ at 15:25:32 UT. Then the burst drifts to higher frequencies ending at 15:25:48 UT as shown in Figure 1. The BSS (Sawant et al. 2001) recorded a group of type-III RS bursts around $2000 \mathrm{MHz}$ having bandwidth of about $500 \mathrm{MHz}$ at 15:33 UT which are superimposed on a continuum as shown in Figure 2.

$U$-type burst: The speed of the exciting agency for the $\sim 6 \mathrm{~s}$ duration descending part of the partial inverted U-type burst can be estimated assuming the original density model of Aschwanden and Benz (1995) suitably modified for high frequencies above $1000 \mathrm{MHz}$ by Meléndez (1997) as given by: $n_{e}=n_{1}\left(\frac{h}{h_{1}}\right)^{-p}$, with $p=5$, $h_{1}=3.5 \times 10^{5} \mathrm{~km}$, and $n_{1}=4.3 \times 10^{7} \mathrm{~cm}^{-3}$. Here $h_{1}$ and $n_{1}$ are the parameters in the transition region from low to high corona where the density law changes from power-law to exponential. The heights of these emissions are estimated for the frequency range of 1000-2000 MHz. Assuming time of flight for the descending part of the partial inverted U-type burst to be $6 \mathrm{sec}$, estimated speeds are $\simeq 4.0 \times 10^{3} \mathrm{~km} / \mathrm{s}$ and $6.5 \times 10^{3} \mathrm{~km} / \mathrm{s}$ at the fundamental and second harmonic, respectively. These speeds are about one order of magnitude less than those speeds of an exciter required to produce type-III bursts and higher than those of an exciter required to produce type-II bursts. Efforts are being made to use $S O H O$ and SXT 


\section{J.R. Cecatto et al.}

data to determine the separation between the footpoints of the flaring loop which will lead us to a better understanding of the type of exciter. Details of this analysis will be published elsewhere.

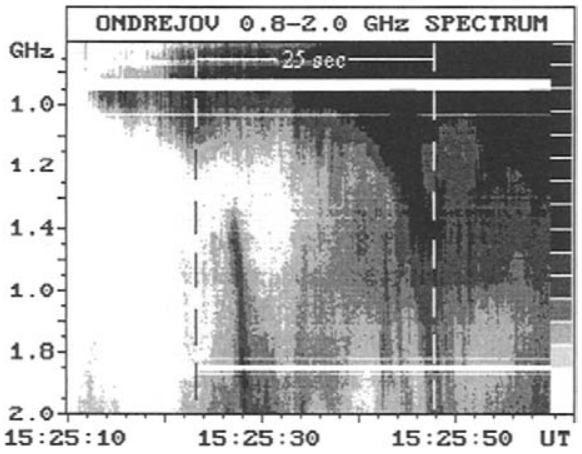

Fig. 1. Radio spectrum (800-2000 $\mathrm{MHz})$ recorded at the Ondrejov Observatory for the April 9th, 2001 event showing a partial inverted U-type burst limited by the dashed lines.

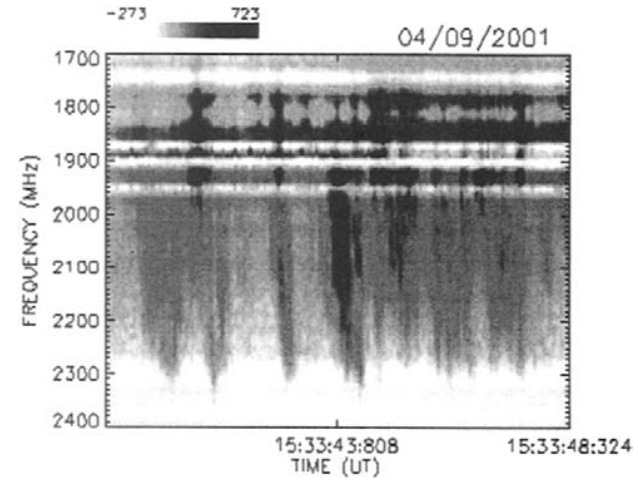

Fig. 2. BSS high resolution spectrum (1700-2400 $\mathrm{MHz}$ ) for the April 9th, 2001 event showing a group of type-III RS bursts.

Type-III RS bursts: The frequency drift rate $D_{f}(\mathrm{MHz} / \mathrm{s})$ is related to the observed frequency $f_{o b s}(\mathrm{MHz})$ as $D_{f}=\frac{f_{o b s}}{2} \frac{V}{H}$. Here $V$ is the speed of the exciting agency in $\mathrm{km} / \mathrm{s}$. For determining the scale height of density variation $H$ we can make use of the same density model as described above. High resolution observations permits us to determine more accurately the drift rates. By using the density model above, the drift rate at high frequencies is given by: $|d f / d t|=(0.09 \pm 0.03) \times f^{(1.35 \pm 0.10)}$ where $d f / d t$ is in $\mathrm{MHz} / \mathrm{s}$, and $\mathrm{f}$ in $\mathrm{MHz}$ (Melendez et al. 1999). Observed drift rates of a sample (15:33:40-15:33:48 UT) of these bursts is in the range of $980-1720 \mathrm{MHz} / \mathrm{s}$, in agreement with the above equation. Thus for $D_{f}=1300 \mathrm{MHz} / \mathrm{s}$ at $f_{o b s}=2 \times 10^{3} \mathrm{MHz}$, we find $V \simeq 2.9 \times 10^{4} \mathrm{~km} / \mathrm{s}$ for type-III RS bursts, which is about one order of magnitude higher than the speed of the exciter determined above for the descending part of the inverted U-type burst.

The decimetric (1000-3000 $\mathrm{MHz}$ ) observations and preliminary analysis of the first reported partial inverted long duration U-type, and high time resolution type-III RS bursts indicate the presence of different exciters. Thus distinct acceleration mechanisms are operating in the flaring region. A more detailed modeling will give more insight into the physics of the flaring region. F.C.R.F., V.K. and M.K. acknowledge scholarship from FAPESP.

\section{REFERENCES}

Aschwanden, M.; A.O. Benz, Chromospheric evaporation and decimetric radio emission in solar flares, Astrophys. J., 438, 997 (1995).

Meléndez, J.L.M. Decimetric solar type-III bursts in association with impulsive phase of solar flares. MSc. Thesis. INPE, São José dos Campos, 1997. 115p. (INPE-6382-TDI/601).

Meléndez, J.L.M; H.S. Sawant, F.C.R. Fernandes, A.O. Benz, Statistical analysis of high-frequency decimetric type III bursts, Solar Phys., 187, 77 (1999).

Sawant, H.S., K.R. Subramanian, C. Faria, F.C.R. Fernandes, J.H.A. Sobral, J.R. Cecatto, R.R. Rosa, H.O. Vats, J.A.C.F. Neri, E.M.B. Alonso, F.P.V. Mesquita, V.A. Portezani, A.R.F. Martinon, Brazilian Solar Spectroscope (BSS), Solar Phys., 200, 167 (2001). 